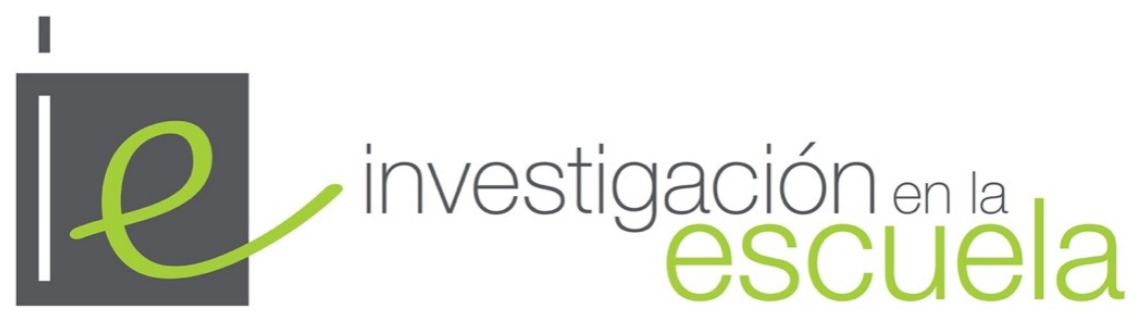

Revista de Investigación e Innovación Educativa n 103, 2021 | e-ISSN 2443-9991

\title{
Huertos ecodidácticos: percepciones sobre formación de profesorado y futuros docentes
}

\author{
The organic learning gardens: perceptions of training by teachers and future teachers
}

iD José Orenes Cárceles es profesor de Educación Primaria en el CEIP Juan XXIII y profesor Asociado en la Facultad de Educación de la Universidad de Murcia (España)·jose.orenes@um.es · https://orcid.org/0000-0001-9852-9444

iD Dr. G. Enrique Ayuso Fernández es profesor Contratado Doctor de la Facultad de Educación de la Universidad de Murcia (España)·ayuso@um.es·https://orcid.org/0000-0002-8510-556X

iD Manuel Fernández Díaz es profesor Asociado en la Facultad de Educación de la Universidad de Murcia (España) · manuel.fernandez2@um.es·https://orcid.org/0000-0001-5956-0035

iD Dr. José María Egea-Fernández es profesor Catedrático de Botánica en la Facultad de Biología de la Universidad de Murcia (España)·jmegea@um.es·https://orcid.org/0000-0001-8323-5259

Cómo citar este artículo

Orenes Cárceles, J., Ayuso, G.E., Fernández Díaz, M. y Egea-Fernández J.M. (2021). Huertos ecodidácticos: percepciones sobre formación de profesorado y futuros docentes. Investigación en la Escuela, 103, 1-18. doi: http://dx.doi.org/10.12795/IE.2021.i103.01

Resumen. La utilización por el profesorado de Ciencias de los huertos escolares sigue en aumento en nuestro contexto al tratarse de un recurso con gran potencial educativo, que permite a los estudiantes poner en práctica el conocimiento escolar, facilita el desarrollo de procesos de investigación, mejora las relaciones entre participantes y es apropiado para el trabajo interdisciplinar. En nuestra investigación hemos analizado la demanda de formación en este campo de docentes en ejercicio y de estudiantes de Grado de Educación Primaria mediante cuestionarios con preguntas cerradas y de tipo Likert. Posteriormente, tras un curso formativo desde el planteamiento de trabajos de indagación, hemos analizado las propuestas llevadas a cabo por los futuros docentes en aulas de Primaria y comparado sus demandas formativas tras sus intervenciones (con un análisis estadístico descriptivo y prueba de significatividad). Los resultados destacan el alto nivel de motivación, la utilidad de la participación activa de escolares y la necesidad de formación específica en aspectos técnicos (técnicas agrícolas, cultivos, semillas, suelos, etc.) y didácticos (uso escolar, actividades, su planificación y evaluación) que desarrollen el currículo, no sólo desde las Ciencias de la Naturaleza, sino desde todas las áreas y que supongan un avance en las competencias del alumnado.

\begin{abstract}
The use by science teachers of school gardens continues to increase in our school context as it is a resource with great educational potential, which allows students to put school knowledge into practice, facilitates the development of research processes, improves relationships between participants and is appropriate for interdisciplinary work. In our research we have analyzed the training needs in this field among practicing teachers and students of the Grade of Primary Education with closed Likert-type questions. After a training based on the approach of enquiry work, we have analyzed the proposals made by future teachers in Primary classrooms and compared their training demands after their interventions (with a descriptive statistical analysis and significance test). The results highlight the high level of motivation, the usefulness of the active participation of schoolchildren and the need for specific training in technical aspects (agricultural techniques, crops, seeds, soils, etc.) and didactics aspects (school use, activity, planning, evaluation) that develop the curriculum, not only from the area of Natural Sciences, but from all areas and that represent an advance in the competences of the students.
\end{abstract}




\section{Palabras clave $\cdot$ Keywords}

Formación inicial del profesorado, procesos científicos, indagación, huertos ecodidácticos. Initial teacher training, scientific processes, inquiry, organic learning gardens.

\section{Introducción y estado de la cuestión}

La utilización en los centros educativos de Infantil, Primaria y Secundaria de los huertos escolares permite al profesorado convertirlos en una lección sobre aspectos de biología en general, ecología e, incluso, un espacio educativo muy valioso para la educación para la sostenibilidad (Larrosa, 2013; Eugenio-Gozalbo et al., 2018; Eugenio-Gozalbo et al., 2019). Son, como se viene aportando por múltiples trabajos, una oportunidad para la implementación en el aula de metodologías activas y experienciales (Eugenio-Gozalbo y Aragón, 2016); en las que el alumnado puede aprender ciencia haciendo ciencia ya que a través de la experimentación pueden desarrollar y adquirir conocimientos, habilidades y actitudes propias de este ámbito del conocimiento (Ceballos et al., 2014; Zuazagoitia et al., 2020). Por todo lo anterior, están suponiendo un incremento en el interés de los centros educativos por disponer de un huerto escolar (Reina et al., 2017).

El trabajo entre el alumnado y profesorado en los huertos escolares permite la realización de actividades prácticas reales basadas en la experiencia, con las que se facilite el aprendizaje de los contenidos curriculares y se pongan en práctica, habilidades propias del trabajo científico como son la observación, la comprobación de ideas, así como percibir y explotar propuestas y actuaciones (Marín-Gil, 2015). Pero, es más, las actividades de enseñanza pueden relacionar el contenido de ciencias con contextos del mundo real, tan relevantes como es la producción de alimentos vegetales, aspecto de gran importancia al poder abordar también el fomento de hábitos saludables de alimentación (Eugenio-Gozalbo et al., 2019).

Dentro de este contexto global, gana peso entre las propuestas llevadas a cabo en los denominados "huertos ecodidácticos" (en adelante HED), la perspectiva agroecológica, con la que se pretende educar en modelos sostenibles de producción, distribución y consumo de alimentos; es decir, más enfocados a la educación para el desarrollo sostenible (Rodríguez et al. 2015; Egea-Fernández et al., 2016; Gutiérrez, 2020; Marín-López, 2020). Así, el uso del HED facilita el trabajo de problemas medioambientales actuales como la pérdida de biodiversidad, la contaminación, el calentamiento global o la inestabilidad del sistema agroalimentario actual, de los que nos interesa conocer sus causas, consecuencias y la forma con la que se puede contribuir a su mitigación (Barrón y Muñoz, 2015; Rekondo et al., 2015). Por otra parte, las actividades diseñadas en el aula, y aplicadas en el huerto del centro, pueden ahondar en el trabajo colaborativo y la utilización de una metodología basada en la indagación al permitir poner en práctica procedimientos relacionados con el método científico (Ceballos et al., 2014), en los que el alumnado establezca conexiones entre el conocimiento adquirido y la realidad, haciéndolo formar parte de una sociedad responsable, crítica y respetuosa con el medio ambiente (Orenes, 2018), lo que permite, además, que el alumnado exprese su talento (Rodríguez-Haros et al., 2013).

Además, el uso del HED ayuda al desarrollo de otras dimensiones del aprendizaje vinculadas a aspectos actitudinales y relacionales (Eugenio-Gozalbo et al., 2019). Así, algunas investigaciones han destacado el aumento de la motivación entre el alumnado al manifestar una disposición positiva hacia la actividad en el huerto y los aprendizajes que conlleva, siendo un recurso que fomenta la implicación del alumnado y su responsabilidad sobre el proceso de aprendizaje (Botella et al., 2017), así como, otros aspectos emocionales como expresar diversión, satisfacción o bienestar por la realización de las actividades (Eugenio-Gozalbo et al., 2019). Entre estos aspectos, no debemos olvidar la importancia que tienen las actividades desarrolladas a través de los HED para abordar temas relativos a los hábitos alimenticios saludables (Rivas, 2017; RamosTruchero et al., 2020) y de consumo responsable, mediante mercadillos en los centros o almuerzos escolares con productos obtenidos de los huertos (Ceballos et al., 2015; Marín-Gil, 2015; Egea-Fernández et al., 2016; Jiménez, 2018) con una deliciosa recompensa: hacer crecer nuestras frutas y verduras con ese sabor de antes que ahora sólo mantienen los productos ecológicos (Muñoz y Yepes, 2020).

Desde otra perspectiva, constituyen un eje transversal para la formación del alumnado, el desarrollo de diversas áreas como Ciencias Naturales, Geografía, Historia, Matemáticas, Tecnología o Lengua (Jiménez, 2018; Egea-Fernández et al., 2016). Más aún, de acuerdo con Llerena (2017), la agroecología escolar impulsa un diálogo entre la escuela y el entorno por lo que puede ser un punto de encuentro con saberes no científicos, locales y una recuperación de la cultura anterior olvidada. Destacamos, que la utilización de los HED, que fomenten las distintas competencias y contribuyen a la difusión de los valores de la agricultura ecológica, puede ayudar a alcanzar los fines establecidos en las legislaciones educativas al incorporar contenidos relacionados con la sostenibilidad medioambiental (LOMLOE, 2020; LOMCE, 2013). Aspecto 
que ha promovido su desarrollo en diferentes resoluciones de programas educativos de promoción de la instalación y uso de huertos escolares en los centros.

Sobre las dificultades acerca de la utilización en los centros de los HED, Egea-Fernández et al. (2014) destaca que a menudo existe una falta de coordinación entre docentes y departamentos para el desarrollo de actividades. Se suele criticar la falta de tiempo para dedicarse a las tareas que requiere (por ejemplo, fuera del calendario académico) o falta de recursos para el cultivo y mantenimiento (Conde et al., 2018). También es importante ser conscientes de la falta de una formación específica, así como de materiales y experiencias curriculares para utilizarlos como recurso de enseñanza (Aragón, 2016). Probablemente, por estas consideraciones algunos estudios indican que, en la mayoría de los casos, solo se emplean como complemento del trabajo del aula, no aprovechando todo su potencial como huertos educativos para conseguir una educación más integral y significativa (Aragón, 2016; Rodríguez y Sánchez, 2018).

En este contexto se inscribe esta contribución: mejorar la formación inicial del profesorado para una utilización más eficiente y completa de este recurso didáctico. Para ello, hemos trabajado con un grupo de estudiantes del último curso del Grado de Educación Primaria que recibieron una formación específica sobre el uso escolar de los huertos ecodidácticos y, a partir de la misma, han planificado, elaborado y aplicado diversas actividades de enseñanza con alumnos y alumnas de Primaria.

\section{Objetivos, muestras y fases del trabajo}

El objetivo principal de nuestro trabajo a largo plazo es el de diseñar, implementar y evaluar un plan de formación adecuado, equilibrado entre teoría y práctica en el diseño, gestión, uso y mantenimiento del HED como recurso educativo para mejorar la formación de los futuros profesores y obtener una utilización más eficiente y completa de este recurso didáctico.

Para poder llevar a cabo este objetivo general, en esta investigación mostramos los siguientes objetivos específicos:

1. En una primera etapa, analizar la opinión y conocimientos de maestros/as en ejercicio del centro en el que se han llevado a cabo las experiencias a través de cuestionarios diseñados para recoger información sobre su opinión acerca del uso escolar de los HED.

2. Analizar los conocimientos iniciales de futuros maestros y maestras en formación estudiantes de $4^{\circ}$ del Grado de Primaria sobre el HED como recurso educativo.

3. Proponer al alumnado del Grado de Primaria un marco metodológico fundamentado en actividades de indagación para el desarrollo de actividades en el HED.

4. Analizar actividades de enseñanza-aprendizaje, diseñadas por estos estudiantes universitarios e implementadas en un colegio con alumnos/as de Primaria utilizando el HED.

5. Analizar la progresión en los conocimientos del alumnado del Grado de Educación Primaria sobre el HED como recurso educativo tras la elaboración y aplicación en el aula de su propuesta educativa.

Nuestras muestras de estudio han estado formadas por dos grupos distintos: profesorado activo de un centro escolar de Primaria y futuro profesorado en formación universitaria.

En el primer caso, se ha tratado de 27 profesores en ejercicio ( 22 maestras y 5 maestros) de un claustro de 59 docentes en total del CEIP Juan XXIII de Murcia, con una experiencia profesional muy heterogénea (desde recién ingresados en la docencia hasta 36 años de experiencia docente). En cuanto a sus estudios de origen, el $60 \%$ estudió bachiller de humanidades, $33 \%$ bachiller de ciencias y un $7 \%$ accedió a magisterio desde Formación Profesional.

Con respecto al profesorado en formación, se ha tratado de 37 alumnos y alumnas de $4^{\circ}$ curso del Grado de Educación Primaria de la Universidad de Murcia que cursaban la asignatura "Talleres de la Naturaleza" dentro de la mención "Recursos educativos para la escuela y el tiempo libre". Estos últimos, que quedaron reducidos a 34 en la fase final, tenían edades comprendidas entre los 21 y 41 años.

El trabajo se llevó a cabo en diversas fases. Las características de cada una se exponen en la Tabla 1. 
Tabla 1

Fases de la implementación de la investigación sobre la utilización del recurso HED entre futuros maestros de Primaria

\begin{tabular}{|c|c|}
\hline Fase & Desarrollo \\
\hline $\begin{array}{l}\text { Evaluación de los } \\
\text { conocimientos } \\
\text { intereses sobre los } \mathrm{HED}\end{array}$ & $\begin{array}{l}\text { De acuerdo con el objetivo 1, se utilizó un cuestionario de conocimientos y } \\
\text { opiniones del profesorado en ejercicio sobre opiniones, intereses y necesidades } \\
\text { formativas acerca de los HED. Fue cumplimentado por los profesores de Primaria } \\
\text { del centro en el que se llevó a cabo la investigación. } \\
\text { Cuestionario inicial de conocimientos y opiniones del alumnado del Grado de } \\
\text { Educación Primaria sobre opiniones, intereses y necesidades formativas acerca de } \\
\text { los HED relacionado con el objetivo } 2 \text {. }\end{array}$ \\
\hline
\end{tabular}

Preparatoria del Formación inicial específica a través de sesiones teórico-prácticas. Metodología alumnado del Grado de participativa en pequeños grupos basada en actividades de indagación siguiendo con Primaria (duración, 3 el objetivo 3.

semanas) Contenidos de la materia seleccionados: a) teóricos y técnicos sobre los HED (cultivos, riego, suelo; compost; semillas; plagas; biodiversidad; cultivos de temporada; o instrumentos agrícolas). También memoria biocultural; planificación y gestión de las tareas del HED; Agroecología y soberanía alimentaria; b) didácticos: ventajas e inconvenientes uso del HED como recurso; diseño e implementación de actividades en el HED; elementos transversales; entre otros.

\begin{tabular}{lrl}
\hline $\begin{array}{l}\text { Diseño e } \\
\text { implementación de }\end{array}$ & $\begin{array}{l}\text { Para alcanzar nuestro objetivo 4, los estudiantes del Grado se distribuyeron en } 9 \\
\text { grupos de trabajo con la finalidad de diseñar e implementar una actividad de } \\
\text { actividades para el HED } \\
\text { enseñanza-aprendizaje en un nivel de Primaria elegido por propia iniciativa, con el }\end{array}$ \\
$\begin{array}{l}\text { en el colegio de Primaria } \\
\text { (duración, 6 semanas) }\end{array}$ & $\begin{array}{l}\text { HED como recurso educativo y con la finalidad del trabajo de diferentes procesos } \\
\text { científicos. Las } 9 \text { actividades planificadas se desarrollaron en el colegio. }\end{array}$ \\
\hline $\begin{array}{l}\text { Evaluación de las } \\
\text { actividades sobre HED }\end{array}$ & $\begin{array}{l}\text { Para lograr nuestro objetivo } 4 \text { se llevó a cabo una evaluación cualitativa de las } \\
\text { actividades llevadas a cabo por los estudiantes del Grado. } \\
\text { implementadas }\end{array}$ \\
& $\begin{array}{l}\text { Cuestionario final alumnos del Grado de Educación Primaria en formación. Con } \\
\text { estructura similar al cuestionario inicial para analizar la evolución de sus } \\
\text { conocimientos acorde con el objetivo 5. }\end{array}$ \\
\hline
\end{tabular}

\section{Material y métodos}

En nuestro trabajo hemos utilizado instrumentos diferentes para cada uno de los objetivos de investigación planteados. De este modo, para conocer la opinión de los maestros y maestras en ejercicio y sus demandas formativas diseñamos y aplicamos un cuestionario de 19 ítems con cuestiones de escala Likert con cuatro niveles (anexo I). En cuanto al estudio del conocimiento práctico sobre los HED por parte de los estudiantes del Grado, diseñamos dos cuestionarios inicial-final con 29 ítems de escala Likert con cuatro niveles (anexo II). Para los análisis cuantitativos de los cuestionarios anteriores utilizamos el programa SPSS versión 24.0.

El cuestionario utilizado para docentes en ejercicio consta de preguntas sobre datos sociométricos y dos dimensiones, la primera relacionada con su experiencia sobre este recurso, con tres preguntas; y la segunda dimensión relativa a su opinión sobre los contenidos necesarios en que se deben formar a los futuros docentes relacionados con el uso, mantenimiento y gestión del HED como recurso educativo con dieciséis preguntas escala Likert (1, no es necesario; 2, es poco necesario; 3 , es necesario; y 4, es muy necesario). Para comprobar la consistencia interna del cuestionario se calculó el alfa de Cronbach que obtuvo un valor de 0,636 para su primera dimensión (dentro del rango aceptable de fiabilidad, de acuerdo con Loewenthal, 2001 y Taber, 2018) En la segunda dimensión el valor del coeficiente fue de 0,860 (evidencia una consistencia interna alta).

Los cuestionarios utilizados con los futuros maestros y maestras, tanto inicial como final, se componen de una parte relativa a datos sociométricos con 5 preguntas cerradas y 2 dimensiones, una relativa a su experiencia y los conocimientos que poseen en el uso, mantenimiento y gestión del HED con 13 preguntas y otra segunda dimensión que recaba su opinión respecto a qué conocimientos consideran necesarios en su formación como futuros docentes para utilizar este recurso con 16 preguntas escala Likert (de 1, no es necesario a 4, es muy necesario). Para la dimensión 1, de este cuestionario, los valores de alfa de Cronbach obtenidos han sido de 0,736 (consistencia interna buena) y de 0,883 para la dimensión 2 (que corresponde 
con una consistencia interna alta). Además, hemos contrastado sus respuestas finales con las iniciales. Para este análisis, hemos realizado un estudio de diferencias significativas a través de una prueba no paramétrica con el estadístico W de Wilconxon, al no suponer la normalidad de las muestras relacionadas y ser la variable dependiente de tipo ordinal (escala Likert) calculando la significancia asintótica bilateral "p" entre dos variables (Gamarra et al., 2020).

Finalmente, se llevó a cabo un análisis cualitativo de las actividades diseñadas e implementadas en el colegio de Primaria en el que se llevaron a cabo las distintas experiencias. Este análisis fue realizado por dos de los autores del trabajo que analizaron por separado los contenidos planteados en las propuestas utilizadas. Posteriormente, se identificaron, de forma consensuada entre ambos, aquellos contenidos trabajados y categorizados como conceptuales, procedimentales y actitudinales en el área de Ciencias de la Naturaleza y otra categoría relativa a contenidos de otras áreas.

\section{Análisis de resultados}

A continuación, describimos los principales resultados de nuestra investigación.

\subsection{Opiniones del profesorado en ejercicio sobre la utilización y necesidades de formación en el recurso HED}

El profesorado en ejercicio del centro en el que se llevó a cabo la puesta en práctica de las propuestas educativas del alumnado en formación del Grado de Primaria respondió a diferentes cuestiones sobre la utilidad de los HED en los centros. En la Tabla 2 mostramos los resultados obtenidos diferenciados por sexos de los participantes y estudios previos a la formación universitaria de los mismos. En esta segregación de datos, hemos querido analizar si en nuestra muestra de profesorado en activo sus opiniones sobre los HED están influidas por estos factores.

Tabla 2

Algunos datos relevantes del profesorado en ejercicio sobre experiencia previa y conocimientos acerca de los HED diferenciado por sexo del docente y estudios previos a su formación universitaria como maestro/ a

\begin{tabular}{lccccc}
\hline Cuestiones & Hombre & Mujer & $\begin{array}{c}\text { Bachillerato } \\
\text { Humanidades }\end{array}$ & $\begin{array}{c}\text { Bachillerato } \\
\text { Ciencias }\end{array}$ & $\begin{array}{c}\text { Formación } \\
\text { Profesional }\end{array}$ \\
\hline $\begin{array}{l}\text { Ha utilizado alguna vez el } \\
\text { recurso educativo HED }\end{array}$ & $2 / 5$ & $14 / 22$ & $11 / 16$ & $4 / 9$ & $1 / 2$ \\
\hline $\begin{array}{l}\text { Considera el HED un recurso } \\
\text { educativo útil }\end{array}$ & $5 / 5$ & $22 / 22$ & $16 / 16$ & $9 / 9$ & $2 / 2$ \\
\hline $\begin{array}{l}\text { Ha recibido formación sobre } \\
\text { HED como recurso educativo }\end{array}$ & $1 / 5$ & $0 / 22$ & $0 / 16$ & $1 / 9$ & $0 / 2$ \\
\hline
\end{tabular}

No apreciamos diferencias debidas al sexo del docente ni a los estudios previos de estos a la hora de manifestar la utilidad del HED como recurso educativo. Igualmente, también al destacar, en su mayor parte, la falta de formación sobre el mismo. Si bien, una mayoría del profesorado indica que lo ha utilizado en alguna ocasión en su ejercicio profesional.

En los datos mostrados en la Tabla 3, comprobamos que independientemente de si han utilizado o no anteriormente el HED, muy mayoritariamente el profesorado en ejercicio considera de gran utilidad la formación tanto en aspectos técnicos como didácticos sobre huertos escolares.

Tabla 3

Algunos datos relevantes del profesorado en ejercicio sobre opiniones acerca de necesidades formativas en HED*

\begin{tabular}{lcc}
\hline Es necesario una formación... & Media & Desv.Típ \\
\hline$\ldots$..especto a los tipos de cultivos a plantar. & 3,70 & 0,465 \\
\hline ...en formas de riego. & 3,63 & 0,492 \\
\hline ...en cuanto a su limpieza, control de plantas no deseadas & 3,67 & 0,480 \\
\hline ...en la realización de compost (compostaje o vermicompostaje) & 3,15 & 1,064 \\
\hline ...sobre las semillas a utilizar & 3,74 & 0,447 \\
\hline
\end{tabular}




\begin{tabular}{lcc}
\hline ...el control de plagas & 3,67 & 0,480 \\
\hline ...la biodiversidad que podemos encontrar en el HED & 3,52 & 0,893 \\
\hline ...la memoria biocultural: saberes y sabores & 3,07 & 1,072 \\
\hline ...planificación y gestión de las tareas del HED & 3,52 & 0,580 \\
\hline ...implementación de actividades en el HED & 3,58 & 0,849 \\
\hline ...cómo abordar los elementos transversales. & 3,63 & 0,565 \\
\hline ...cómo abordar contenidos del área de Ciencias de la Naturaleza. & 3,81 & 0,396 \\
\hline ...cómo abordar contenidos del resto de áreas del currículo de Primaria. & 3,48 & 0,509 \\
\hline ...las ventajas del uso del HED como recurso educativo. & 3,52 & 0,509 \\
\hline ...los inconvenientes del uso del HED como recurso educativo. & 3,00 & 1,000 \\
\hline ...HED y los principios de la Agroecología & 3,22 & 0,641 \\
\hline${ }^{*}$ Media y desviación típica obtenidas de valores de la escala Likert (1. No es necesario. 2. Es poco necesario. 3. Es necesario y 4. Es muy necesario).
\end{tabular}

La mayor parte de los docentes destacan la necesidad de recibir formación específica sobre asuntos como tipos de cultivos, semillas, compostaje, plagas, biodiversidad, memoria biocultural y, especialmente, planificación e implementación de actividades relacionadas con los contenidos didácticos del área de Ciencias de la Naturaleza, de otras áreas y de los contenidos transversales con este recurso.

\subsection{Experiencias, conocimientos y opiniones iniciales y finales sobre el recurso HED de los futuros maestros y maestras de Primaria}

En la Tabla 4 se recoge un resumen de sus experiencias iniciales en el uso del HED desde la perspectiva del alumnado que ha podido ser formado con este recurso educativo, como desde el punto de vista de docentes en formación que lo podrían utilizar. También añadimos, para un análisis comparativo, las respuestas a las mismas cuestiones tras sus intervenciones didácticas con el alumnado de Primaria.

Tabla 4

Algunos datos relevantes del cuestionario inicial y final del alumnado del Grado de Educación Primaria sobre sus experiencias en el uso del HED como recurso educativo

\begin{tabular}{lcc}
\hline Cuestiones & $\begin{array}{c}\text { Inicial } \\
\text { (N=37) }\end{array}$ & $\begin{array}{c}\text { Final } \\
(\mathbf{N}=\mathbf{3 4})\end{array}$ \\
\hline Ha utilizado alguna vez el recurso educativo HED & 1 & 34 \\
\hline Considera que el HED es un recurso educativo útil en Educación Primaria & 37 & 34 \\
\hline Conoce alguna ventaja educativa del uso del recurso HED & 29 & 34 \\
\hline Conoce algún inconveniente del uso del recurso HED & 13 & 15 \\
\hline $\begin{array}{l}\text { Ha recibido formación sobre el diseño, gestión, uso y mantenimiento del } \\
\text { HED como recurso educativo }\end{array}$ & 3 & 34 \\
\hline $\begin{array}{l}\text { Considera que la formación que ha recibido es suficiente para el diseño, } \\
\text { gestión, uso y mantenimiento del HED como recurso educativo }\end{array}$ & 0 & 25 \\
\hline
\end{tabular}

De los 37 estudiantes del Grado de Primaria, al inicio de nuestro trabajo, sólo uno había utilizado previamente el HED y todos afirmaron que es un recurso útil en Primaria. Aunque son muchos, 29 en total, los que señalan que el HED ofrece muchas ventajas educativas; algunos, 13 de los 37 , indican que su uso se dificulta por sus inconvenientes.

La formación posterior recibida por los estudiantes (en la que participaron 34 de los 37) que abordó contenidos didácticos sobre este recurso, así como una formación teórico-práctica (creando el huerto y plantando distintos cultivos pues se realizó una plantación de invierno en los huertos de la Facultad de Biología) junto con el diseño e implementación en las aulas de Primaria de actividades de enseñanzaaprendizaje relacionadas con el uso del huerto escolar, ha reforzado, en nuestra opinión, su valoración de la utilidad del HED como recurso educativo y las ventajas de uso. Asimismo, identificando nuevas desventajas, derivadas de la experiencia práctica, y resaltando la importancia de la formación recibida. En relación a su formación sobre contenidos teóricos, prácticos y didácticos, en la Tabla 5 mostramos algunos datos que consideramos de interés. 
Tabla 5

Algunos datos relevantes del cuestionario inicial y final del alumnado del Grado de Educación Primaria sobre su formación en contenidos teóricos, prácticos y didácticos del HED como recurso educativo

\begin{tabular}{lcc}
\hline Cuestiones & $\begin{array}{c}\text { Inicial } \\
\text { (N=37) }\end{array}$ & $\begin{array}{c}\text { Final } \\
\text { (N=34) }\end{array}$ \\
\hline $\begin{array}{l}\text { Conoce alguno de los cultivos que se pueden plantar en un HED en otoño } \\
\text { o en primavera }\end{array}$ & 8 & 27 \\
\hline $\begin{array}{l}\text { Conoce alguna de las plantas no deseadas que hay que controlar o eliminar } \\
\text { en un HED }\end{array}$ & 9 & 18 \\
\hline Conoce cómo se obtiene compost & 11 & 19 \\
\hline Conoce cómo se gestiona un banco de semillas para un HED & 0 & 4 \\
\hline Conoce alguna plaga habitual en los HED & 11 & 24 \\
\hline Conoce algún principio de la Agroecología & 4 & 3 \\
\hline Sabe en qué consiste la soberanía alimentaria & 0 & 1 \\
\hline
\end{tabular}

$\mathrm{Al}$ inicio del trabajo, ninguno de los estudiantes de nuestro estudio mostró conocimientos sobre la gestión de bancos de semillas o el significado de la soberanía alimentaria y muy pocos reconocían algún principio de agroecología. Una vez finalizadas las intervenciones, sin embargo, se aprecia una mejora importante en su formación en aspectos prácticos como cultivos, plagas, gestión de plantas no deseadas y obtención de compost. No obstante, se constata que sigue siendo necesaria más formación sobre la gestión de semillas, que requiere una intervención específica, los principios de la agroecología o la soberanía alimentaria.

Por otra parte, también analizamos antes y después de la intervención con los estudiantes universitarios, sus opiniones sobre diversos aspectos formativos acerca del diseño, gestión, uso y mantenimiento del HED. En la Tabla 6 se recoge la valoración de los aspectos formativos sugeridos por parte de los estudiantes de nuestra muestra, así como un estudio de significatividad de las diferencias.

Tabla 6

Datos más relevantes del cuestionario inicial y final del alumnado del Grado de Educación Primaria sobre sus opiniones en aquellos contenidos formativos sobre diseño, gestión, uso y mantenimiento del HED*

\begin{tabular}{|c|c|c|c|c|c|}
\hline \multirow[t]{2}{*}{ Es necesario formar... } & \multicolumn{2}{|c|}{ Inicial } & \multicolumn{2}{|c|}{ Final } & \multirow{2}{*}{$\begin{array}{l}\text { Sig. asintót. } \\
\text { (biateral) }\end{array}$} \\
\hline & Media & Desv.Tip & Media & Desv.Tip & \\
\hline $\begin{array}{l}\text {...respecto a los tipos de HED y sus } \\
\text { diseños }\end{array}$ & 3,65 & 0,538 & 3,74 & 0,511 & 0,454 \\
\hline $\begin{array}{l}\text {...respecto a los tipos de cultivos a } \\
\text { plantar. }\end{array}$ & 3,59 & 0,551 & 3,74 & 0,511 & 0,282 \\
\hline $\begin{array}{l}\text {...en cuanto a su limpieza, control de } \\
\text { plantas no deseadas }\end{array}$ & 3,59 & 0,599 & 3,74 & 0,448 & 0,244 \\
\hline $\begin{array}{l}\text {...en la realización de compost } \\
\text { (compostaje o vermicompostaje) }\end{array}$ & 3,11 & 1,073 & 3,56 & 0,615 & 0,026 \\
\hline ...sobre las semillas a utilizar & 3,58 & 0,554 & 3,68 & 0,535 & 0,326 \\
\hline ...el control de plagas & 3,62 & 0,594 & 3,74 & 0,448 & 0,285 \\
\hline $\begin{array}{l}\text {...la biodiversidad que podemos } \\
\text { encontrar en el HED }\end{array}$ & 3,62 & 0,594 & 3,62 & 0,551 & 0,827 \\
\hline $\begin{array}{l}\text {...la memoria biocultural: saberes y } \\
\text { sabores }\end{array}$ & 3,35 & 0,538 & 3,50 & 0,508 & 0,221 \\
\hline $\begin{array}{l}\text {...planificación y gestión de las tareas } \\
\text { del HED }\end{array}$ & 3,35 & 0,633 & 3,41 & 0,605 & 0,655 \\
\hline $\begin{array}{l}\text {...implementación de actividades en el } \\
\text { HED }\end{array}$ & 3,43 & 0,603 & 3,29 & 0,836 & 0,614 \\
\hline $\begin{array}{l}\text {...cómo abordar los elementos } \\
\text { transversales. }\end{array}$ & 3,59 & 0,798 & 3,59 & 0,500 & 0,467 \\
\hline $\begin{array}{l}\text {...cómo abordar contenidos del área de } \\
\text { Ciencias de la Naturaleza. }\end{array}$ & 3,57 & 0,502 & 3,53 & 0,563 & 0,808 \\
\hline $\begin{array}{l}\text {...cómo abordar contenidos del resto } \\
\text { de áreas del currículo de Primaria. }\end{array}$ & 3,38 & 0,639 & 3,53 & 0,563 & 0,221 \\
\hline
\end{tabular}




\begin{tabular}{lccccc}
\hline $\begin{array}{l}\text {..las ventajas del uso del HED como } \\
\text { recurso educativo. }\end{array}$ & 3,43 & 0,588 & 3,59 & 0,500 & 0,251 \\
\hline $\begin{array}{l}\text {...los inconvenientes del uso del HED } \\
\text { como recurso educativo. }\end{array}$ & 3,35 & 0,588 & 3,56 & 0,613 & 0,290 \\
\hline $\begin{array}{l}\text {..el HED y los principios de la } \\
\text { Agroecología }\end{array}$ & 3,08 & 0,954 & 3,35 & 0,544 & 0,117 \\
\hline
\end{tabular}

*Media, desviación típica y significancia asintótica bilateral obtenidas de valores de la escala Likert (1. No es necesario. 2. Es poco necesario. 3. Es necesario y 4. Es muy necesario).

Del análisis de los datos, deducimos la necesidad de formación inicial en todos los aspectos abordados; si bien, aquellos que están más relacionados con aspectos didácticos, como la planificación y gestión de tareas, la implementación de actividades, cómo abordar los contenidos de otras áreas distintas a las ciencias; o que tienen un carácter más general sobre los HED (ventajas e inconvenientes de su uso, por ejemplo) requieren una demanda formativa ligeramente inferior (valores menores de 3,43) a otros de carácter más específico en relación con la gestión de los huertos. Así, los aspectos más técnicos relativos al mantenimiento y uso del huerto día a día requieren más formación (valores cercanos a 4), especialmente en aspectos como el control de plagas, los tipos de cultivos a emplear, la limpieza y control de plantas no deseadas, el uso de semillas o el estudio de la biodiversidad en los mismos.

Siguiendo con nuestro análisis pretest-postest, tras el periodo formativo teórico-práctico y el diseño e implementación de actividades, los futuros docentes participantes consideran mayoritariamente que los contenidos más necesarios en su formación son: tipos y diseño de HED, cultivos, control de plantas no deseadas, semillas y control de plagas; tanto teoría como práctica; en la fase final de formación aumentan su porcentaje reforzando sus opiniones respecto a su relevancia. En cuanto a su formación en contenidos didácticos los consideran muy necesarios: implementación de actividades, los temas transversales, los contenidos del área de Ciencias de la Naturaleza y del resto de áreas; respecto a ventajas e inconvenientes del uso de este recurso aumentaron tras el proceso de formación debido, probablemente, al desconocimiento que tenían inicialmente del mismo.

Por otra parte, sólo detectamos diferencia significativa en sus opiniones iniciales y finales en el ítem: "es necesario formar en la realización de compost (compostaje o vermicompostaje)" pues se obtiene un valor menor que 0,05 . Respecto al resto de ítems, no existen diferencias significativas entre sus opiniones iniciales y finales, aunque sí consideran, tanto al inicio como al final de esta investigación, que son cuestiones importantes para su formación el diseño, uso y gestión de los HED (con medias por encima de 3). De este modo, consideramos que su predisposición a recibir dicha formación siempre ha sido valorada como necesaria o muy necesaria desde el conocimiento y la implementación didáctica de la misma ha servido para su confirmación.

\subsection{Análisis de las actividades de enseñanza sobre el HED diseñadas por los futuros maestros y maestras}

La mayor parte de las propuestas implementadas por los estudiantes del Grado en el centro de Primaria trataron contenidos del currículo del área de Ciencias de la Naturaleza. Solo dos abordaron actividades con carácter interdisciplinar con áreas como la Lengua o la Plástica. Las actividades fueron evaluadas, en una primera etapa por el profesorado en ejercicio del centro responsable de los distintos cursos; y posteriormente, por los autores de esta comunicación. En la Tabla 7 mostramos un resumen de los contenidos abordados y su frecuencia.

Tabla 7

Análisis de las actividades diseñadas, contenidos trabajados y procesos científicos llevados a cabo por los estudiantes del Grado de Educación Primaria

Contenidos abordados en las distintas actividades

Frecuencia $\mathbf{N}=9$

Cultivos, plantas del HED

Biodiversidad: flora y fauna

Contenidos

Semillas: obtención, clasificación, conservación

relativos a

Herramientas: normas de utilización y protección 


\begin{tabular}{|c|c|c|}
\hline \multirow{10}{*}{$\begin{array}{c}\text { Contenidos } \\
\text { relativos a } \\
\text { procedimientos }\end{array}$} & Observar & 9 \\
\hline & Clasificar & 8 \\
\hline & Experimentar & 3 \\
\hline & Comunicar & 3 \\
\hline & Predecir & 2 \\
\hline & Identificar variables & 2 \\
\hline & Emitir hipótesis & 2 \\
\hline & Medir & 2 \\
\hline & Analizar datos & 2 \\
\hline & Establecer conclusiones & 2 \\
\hline \multirow{4}{*}{$\begin{array}{l}\text { Contenidos } \\
\text { relativos a } \\
\text { actitudes }\end{array}$} & Cuidado del Medio Ambiente & 9 \\
\hline & Respeto a los compañeros & 5 \\
\hline & Trabajo en equipo & 4 \\
\hline & Seguridad y cuidado en el uso de herramientas & 1 \\
\hline \multicolumn{2}{|c|}{ Contenidos de otras áreas (Lengua y Plástica) } & 2 \\
\hline
\end{tabular}

Los contenidos conceptuales más trabajados en las actividades diseñadas e implementadas por los alumnos de Grado fueron: tipos de cultivos y semillas que utilizaron para plantar, la biodiversidad (plantas y animales del HED), observar y clasificar objetos, seres vivos y fenómenos en el HED y el cuidado del medio ambiente, trabajo colaborativo y respeto de las ideas de los compañeros.

En todas las actividades diseñadas se abordaron desde el área de Ciencias de la Naturaleza elementos transversales como Educación ambiental, Educación moral y cívica y Educación para la paz y convivencia. En cuanto a contenidos procedimentales, algunos procesos científicos apenas si se trataron en la implementación de las actividades, entre ellos: medir, identificar variables, predecir, emitir hipótesis, analizar datos y establecer conclusiones. Por otro lado, entre los contenidos actitudinales más destacados, en todas las actividades se trabajó el cuidado del Medio Ambiente, debido posiblemente al trabajo en contacto con los seres vivos que conforman un HED y en muchas, respeto hacia los compañeros y el trabajo colaborativo. Por último, el trabajo interdisciplinar sólo se realizó en dos actividades donde se trataron contenidos del área de Lengua (al crear un cuento y una noticia) y de Artística (al confeccionar un espantapájaros temático)

Tanto el alumnado de Primaria como sus tutoras manifestaron satisfacción en las tareas implementadas y solicitaban que fuese su grupo donde se llevasen a cabo. Además, las actividades diseñadas en el aula, y aplicadas en el huerto del centro, mediante el trabajo colaborativo y la utilización de una metodología basada en actividades de indagación permitieron poner en práctica procedimientos relacionados con el método científico.

\section{Discusión y conclusiones}

Las valoraciones realizadas por los estudiantes del Grado de Primaria, así como, las del profesorado en ejercicio que han conocido las actividades realizadas en el centro escolar, muestran un elevado nivel de satisfacción con la utilización del recurso HED, al igual que se ha evidenciado por otras investigaciones (Eugenio-Gozalbo et al., 2019, por ejemplo). A partir de la información recogida sobre las opiniones de estudiantes universitarios y maestros/as y del análisis de las actividades puestas en práctica, podemos establecer al menos tres conclusiones principales:

Tanto los futuros docentes como el profesorado en activo han manifestado la necesidad de formación en HED (Rodríguez y Sánchez, 2018). Esta necesidad se repite en muchos centros educativos donde los docentes demandan una formación didáctica (uso escolar, actividades, planificación de las mismas, evaluación, etc.) que les permita conocer propuestas ya diseñadas para implementar y saber diseñar sus propias actividades más apropiadas a su alumnado y entorno (Aragón, 2016).

El uso educativo del HED, puede permitir al profesorado el trabajo de competencias relacionadas con los procesos científicos con el alumnado de Primaria, como es la realización de observaciones, clasificaciones o mediciones, la realización de predicciones o la identificación de variables, entre otros muchos (de forma similar a lo expuesto en otros trabajos como el de Ceballos et al. (2014).

En nuestra investigación se destaca la necesidad formativa en aspectos de carácter más técnico como la gestión de bancos de semillas, técnicas agrícolas, suelos, el significado de la soberanía alimentaria o los principios de la agroecología, entre otros (coincidiendo con Egea-Fernández et al., 2016; Melo, 2019). Igualmente, el alumnado pudo comprobar la importancia de producir compostaje como abono para los 
cultivos y como medio de aprovechar recursos naturales del HED utilizando la biomasa que generan las plantas no deseadas, podas o restos y otros residuos orgánicos que se producen en los colegios; mientras que al inicio del proceso de formación recibido no conocían este aspecto técnico tan vinculado a la experiencia práctica (Eugenio-Gozalbo et al., 2020).

Respecto a cómo trabajar en los HED para poder lograr el cumplimiento de los máximos objetivos, encontramos que la investigación escolar, con propuestas constructivistas (Morales et al., 2017), podría ser un marco de referencia en el que transitar en este sentido. En este aspecto, como señala Castaneda (2019) y Eugenio-Gozalbo et al. (2018), consideramos muy conveniente la formación en una metodología que propicie las actividades de indagación por los escolares de modo que se puedan poner en práctica los procesos que caracterizan la actividad de las ciencias.

Al mismo tiempo, consideramos de gran utilidad para el alumnado de Primaria la producción de frutas y verduras por sus propios medios, con frecuencia lejos de su experiencia cotidiana; además de la realización de trabajos al aire libre, compartiendo recursos naturales posibilitando el trabajo cooperativo y mejorando las relaciones entre niños y niñas como indican Castaneda (2019), Jurado (2017), Ramos (2017) y Sántiz (2018), al mismo tiempo que representan una oportunidad para la convivencia, colaboración y encuentro entre la comunidad escolar (Armienta et al., 2019). También aquí, consideramos conveniente la existencia del coordinador del HED en los centros escolares como figura formada, con disponibilidad temporal y acceso a recursos para asesorar a los diferentes docentes en todas las labores y actividades que en él se desarrollen, como señalan Espinet y Llerena (2013) y Egea-Fernández et al. (2016).

Finalmente, somos conscientes de la necesidad de seguir profundizando en la formación inicial del profesorado en el HED y de poner en prácticas más ejemplos de actividades diseñadas y programadas para desarrollar el currículo, no sólo desde Ciencias de la Naturaleza sino desde todas las áreas, de modo interdisciplinar y transversal, que puedan servir de modelo a los docentes y que faciliten el diseño de otras propias mejor adaptadas a su contexto educativo.

\section{Referencias}

Aragón, L. (2016). El huerto ecológico como elemento innovador en educación Ambiental para la formación inicial de maestros/as. En EDUNOVATIC2016. I Congreso Virtual internacional de Educación, Innovación y TIC, 276-278. http:/ / bit.ly/3b3fJu6

Armienta, D. E., Keck, C., Ferguson, B. G. y Saldívar, A. (2019). Huertos escolares como espacios para el cultivo de relaciones. Innovación. Educativa, 19(80), 161-178. https://bit.ly/3r51Tg9

Barrón, Á. y Muñoz, J. M. (2015). Los huertos escolares comunitarios: fraguando espacios socioeducativos en y para la sostenibilidad. Foro de Educación, 13(19), 213-239. http://dx.doi.org/10.14516/fde.2015.013.019.010

Botella, A. M., Hurtado, A. y Cantó, J. (2017). El huerto escolar como herramienta innovadora que contribuye al desarrollo competencial del estudiante universitario. Una propuesta educativa multidisciplinar. Vivat Academia Revista de comunicación, 139, 31. https://doi.org/10.15178/va.2017.139.19-31

Castaneda, M. (2019). El huerto escolar como herramienta pedagógica para la asignatura de Ciencia Salud y Medio Ambiente en el Tercer ciclo del Centro Escolar Instituto Nacional de Santa Ana. Conocimiento Educativo, 6, 69-85. https://doi.org/10.5377/ce.v6i0.8042

Ceballos, M., Escobar, T. y Vílchez, J. E. (2014). El huerto escolar: percepción de futuros maestros sobre su utilidad didáctica. En Actas XXVI Encuentros de Didáctica de las Ciencias Experimentales, $285-292$. https: / / bit.ly/37T4Usm

Conde, M. C., Mariscal, C. y Cepeda, J. (2018). La metodología en el trabajo de huerto escolar y coherencia con la ambientalización curricular. Análisis de una práctica docente. Didáctica de las Ciencias Experimentales y Sociales, 35, 113-126. https://doi.org/10.7203/dces.35.12799

Egea-Fernández, J. M., Egea, J. M. y Valera, M. (2014). Huertos escolares ecológicos de la región de Murcia. XI Congreso de SEAE: «Agricultura ecológica familian», 1-18. https://bit.ly/3suMANX

Egea-Fernández, J. M., Egea, J. M. y Guerrero, M. (2016). Huertos Escolares Como Recurso Para La Educación Agroecológica. El Caso De La Región De Murcia. Agroecología, 11(1), 19-29. https://bit.ly/2ZVJ8jd

Espinet, M. y Llerena, G. (2013). Un análisis exploratorio de narrativas sobre la experiencia en un programa de formación ocupacional en agroecología escolar comunitaria. Enseñanza de las ciencias. revista de investigación y experiencias didácticas, 1137-1141. https://bit.ly/3ea2eZU 
Eugenio-Gozalbo, M. y Aragón, L. (2016). Experiencias en torno al huerto ecológico como recurso didáctico y contexto de aprendizaje en la formación inicial de maestros de Infantil. Revista Eureka sobre Enseñanza Divulgación De Las Ciencias, 13(3), 667-679. https://doi.org/10498/18504

Eugenio-Gozalbo M., Zuazagoitia D. y Ruiz-González A. (2018). Huertos EcoDidácticos y Educación para la Sostenibilidad. Experiencias educativas para el desarrollo de competencias del profesorado en formación inicial. Revista Eureka sobre Enseñanza y Divulgación de las Ciencias, 15(1), 1501. https://doi.org/10.25267/Rev_Eureka_ensen_divulg_cienc.2018.v15.11.1501

Eugenio-Gozalbo M., Vílchez López J. E., Aragón Núñez L. y Ceballos Aranda M. (2018). ¿Qué percepciones tienen los maestros en formación inicial sobre las estrategias y contenidos que aprenden en el huerto ecodidáctico? 28 Encuentros de Didáctica de las Ciencias Experimentales: iluminando el cambio educativo, 403-408. https://bit.ly/2RB7JJr

Eugenio-Gozalbo, M., Ramos Truchero, G. y Vallés Rapp, C. (2019). Huertos universitarios: dimensiones de aprendizaje percibidas por los futuros maestros, Enseñanza de las ciencias, 37(3), 111-127. https://doi.org/10.5565/rev/ensciencias.2657

Eugenio-Gozalbo, M., Ramos Truchero, G. \& Suárez López R. (2020). University gardens for sustainable citizenship: assessing the impacts of garden-based learning on environmental and food education at Spanish Higher Education. International Journal of Sustainability in Higher Education, 22(3), 516-534. https://doi.org/10.1108/IJSHE-06-2020-0208

Gamarra, G., Pujay, O. E. y Ventura, M. (2020). Aplicación de las pruebas estadísticas de Wilcoxon y MannWhitney con SPSS. Revista De Investigación Multidisciplinaria CTSCAFE, 2(4), 15. https://bit.ly/3a4huWX

Gutiérrez, M. L. (2020). El huerto escolar: una herramienta pedagógica para la conciencia medioambiental del alumnado. Revista de educación, innovación y formación, 2, 43-61 https://bit.ly/3tg55qx

Jiménez, D. (2018). Todo empieza con una semilla. Aula de Secundaria, 15, 34-39. https://bit.ly/3e1Qwlk

Jurado, M. A. (2017). El Huerto escolar como estrategia pedagógica para el fortalecimiento de competencias ciudadanas en estudiantes del grado quinto en la Institución Educativa Pablo Correa León sede Guillermo León V alencia. (Tesis de Maestría en Educación). Universidad Autónoma de Bucaramanga. https://bit.ly/2MxQLcN

Larrosa, F. J. (2013). Huertos escolares de la Región de Murcia. (Proyecto Fin de Carrera. Licenciatura de Ciencias Ambientales). Universidad de Murcia. https://bit.ly/3uBTnYe

Llerena, G. (2017). Estudio de caso sobre la colaboración entre la escuela y la comunidad para el desarrollo de la Agroecología Escolar. Enseñanza de las ciencias, Extra, 3099-3104. https://bit.ly/3kvwGAa

LOMCE (2013). Ley Orgánica 8/2013, de 9 de diciembre, para la mejora de la calidad educativa. Boletín Oficial del Estado, 295, de 10 de diciembre de 2013, 97858-97921. https://bit.ly/3sksghO

LOMLOE (2020). Ley Orgánica 3/2020, de 29 de diciembre, por la que se modifica la Ley Orgánica 2/2006, de 3 de mayo, de Educación. Boletín Oficial del Estado, 340, de 30 de diciembre de 2020, 122868-122953. https://bit.ly/3mN3INu

Loewnthal, K. M. (2001). An introduction to psychological tests and scales. 2nd edition. UCLPress. https://bit.ly/3goqslJ

Marín-Gil, J. (2015). El huerto escolar: mágica fuente de conocimiento y sabiduría. Aula, 239, 44-47. http://bit.ly/3bKaoqw

Marín-López, B. (2020). El consumo responsable a través del buerto escolar: propuesta didáctica para educación primaria. (Trabajo de Fin de Grado Educación Primaria). Universidad de Ávila. http://bit.ly/3b1t7i7

Melo, M. P. (2019). Reflexiones sobre el uso de huertas escolares como propuestas pedagógicas y didácticas para el abordaje de la problemática y seguridad alimentaria en el nivel escolar de básica secundaria. Biografía. Escritos sobre la Biología y su Enseñanza. Edición Extraordinaria. Memorias del X Encuentro Nacional de Experiencias en Enseñanza de la Biología y la Educación Ambiental. $V$ Congreso Nacional de Investigación en Enseñanza de la Biologia, 235-245. http://bit.ly/3syqme1

Morales, E., Rodríguez, M. y Parra, G. (2017). Diseño e implementación de un buerto universitario ecológico como recurso didáctico para futuros maestros de Educación Primaria e Infantil (Campus Viriato, Zamora) ID2016/045. Programa de mejora de la calidad-Plan estratégico general 2013-2018. (Memoria Final del Proyecto de Innovación y mejora docente). Universidad de Salamanca. https://bit.ly/3kuom3T

Muñoz, O. M. y Yepes, A. (2020). Elaboración de compostaje con los residuos orgánicos del comedor para el huerto escolar ecológico. Revista de educación, innovación y formación, 2, 123-136 https://bit.ly/3ktLZcV

Orenes Cárceles, J. (2018). Proyecto Huerto Escolar Ecológico en el CEIP Puente de Doñana (no publicado). 
Ramos Truchero, G., Eugenio Gozalbo, M. y Suárez-López, R. (2020). Los huertos ecodidácticos como espacios de formación para una ciudadanía alimentaria. Consumo Socialmente Responsable y Gobernanza Alimentaria. Casos prácticos docentes, 115-128. https:/ / bit.ly/3toaGLc

Ramos, P. (2017). Huerto Escolar como centro de aprendizaje. (Trabajo de Fin de Grado de Educación Primaria). Universidad de las Palmas de Gran Canaria. http://bit.ly/37Rh7xK

Reina, M., Vílchez, J. E., Ceballos, M. y López, J. M. (2017). Análisis de un proyecto de huerto escolar en secundaria a partir de las percepciones de los estudiantes. Enseñanza de las ciencias: revista de investigación y experiencias didácticas. Extra, 1491-1496. https://bit.ly/3kv58Lr

Rekondo, M., Espinet, M. y Llerena, G. (2015). La construcción discursiva de la competencia eco-ciudadana en la escuela: La realización de un diseño tecnológico colaborativo en agroecología escolar. Investigación en la escuela, 86, 7-19. http:/ / dx.doi.org/10.12795/IE.2015.i86.01

Rivas, N. D. V. (2017). Huerto Escolar como Estrategia Pedagógica de la Sustentabilidad en la Educación Ambiental. (Proyecto en ejecución). Revista Scientific, 2, 355375.https://doi.org/10.29394/scientific.issn.2542-2987.2017.0.0.19.355-375

Rodríguez-Haros, B., Tell-García, E. y Aguilar-Californias, S. (2013). Huerto escolar: estrategia educativa para la vida. Ra Ximhai, 9(1), 25-32. http://bit.ly/3pZL9p9

Rodríguez, F., Fernández, J. y García, J.E. (2015). El huerto escolar ecológico como herramienta para la educación en y para el decrecimiento. Investigación en la escuela, 86, 35-48, doi: https://doi.org/10.12795/IE.2015.i86

Rodríguez, B. y Sánchez, L. (2018). ¿Es el huerto escolar ecológico una propuesta didáctica presente en las escuelas infantiles (0-3 años) de la provincia de Sevilla? (Trabajo Fin de Grado en Educación Primaria). Universidad de Sevilla. https://bit.ly/2P19rgX

Sántiz, G. (2018). El huerto escolar, oportunidad para fortalecer el trabajo colaborativo y la integración entre los estudiantes de escuelas primarias. (Tesis de maestría) El Colegio de la Frontera Sur, San Cristóbal de Las Casas, México. http://bit.ly/3b1HrHB

Taber, K. S. (2018). The Use of Cronbach's Alpha When Developing and Reporting Research Instruments in Science Education. Research in Science Educación, 48, 1273-1296. http://doi.org/10.1007/s11165-016$9602-2$

Zuazagoitia, D., Aragón, L., Ruiz, A., Eugenio-Gozalbo, M. y Rodríguez, F. (2019). El desarrollo de la competencia científica del profesorado en formación inicial en el contexto de los huertos ecodidácticos: diseño de una secuencia didáctica en torno al suelo y la ciencia ciudadana. BOLETÍN ENCIC. Revista del Grupo de Investigación HUM-974, 3(2), 138-141. https://bit.ly/3e8Tirq 
Anexo I. Cuestionario del profesorado en ejercicio sobre opiniones, intereses y necesidades formativas acerca de los HED

\section{CUESTIONARIO MAESTROS/AS: EL HUERTO ESCOLAR ECOLÓGIO (HED) COMO RECURSO EDUCATIVO}

Vamos a realizar una investigación sobre la formación necesaria para el DISEÑO, USO y MANEJO del HED como RECURSO EDUCATIVO de nuestro alumnado de la mención de Recursos de $4^{\circ}$ Grado en Primaria.

Para conocer cuál debe de ser la formación necesaria y así poder realizar un diseño adecuado necesito de vuestra colaboración cumplimentando un cuestionario anónimo sobre cuáles van a ser los contenidos necesarios sobre los que trabajar con nuestros estudiantes de la mención de Recursos partiendo de vuestra opinión como maestros/as.

Este cuestionario se compone de dos partes, una relativa a datos sociométricos y su experiencia y opinión sobre este recurso, el HED; y otra relativa a su opinión sobre los contenidos necesarios en que se deben formar a los futuros docentes relacionados con el uso, mantenimiento y gestión del HED como recurso educativo. Cumplimentarlo no le llevará más de 4 minutos, gracias por su atención.

1.- Datos sociométricos:

Sexo Hombre $\square$ Mujer $\square$

Edad $\square$ Años de experiencia

Estudios previos de acceso al Grado de Magisterio:

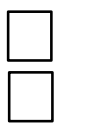

Bachiller de Ciencias

Bachiller de Humanidades y C. Sociales

Bachiller de Artes

Formación Profesional

2.- ¿Ha utilizado alguna vez el recurso educativo HEE?

SI

$\mathrm{NO}$

3.- Considera que el HED es un recurso educativo útil.

SI

NO $\square$

4.- ¿Ha recibido formación sobre el HED como recurso educativo?

SI $\square$ NO

CUESTIONARIO MAESTROS/AS. CONTENIDOS NECESARIOS EN LA FORMACIÓN DE LOS FUTUROS DOCENTES SOBRE EL USO, GESTIÓN Y MATENIMIENTO DEL HED COMO RECURSO EDUCATIVO.

Señale desde 1 a 5 la opción que usted considera más adecuada utilizando los siguientes valores:

\section{1.- No es necesario. 2.- Es poco necesario. 3. Es necesario y 4.- Es muy necesario.}

1.- Es necesario formar respecto a los tipos de cultivos a plantar.

2.- Es necesario formar en formas de riego.
4<smiles>C1CCC1</smiles>

1

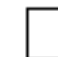

2

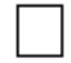

3

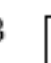

4 
3.- Es necesario formar en cuanto a su limpieza, control de plantas no deseadas.
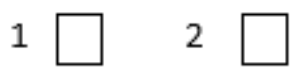

3

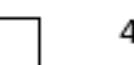

4. Es necesario formar en la realización de compost tanto en compostaje como en vermicompostaje.

1

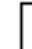
2

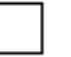

3
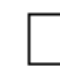

5.- Es necesario formar sobre las semillas a utilizar.

1
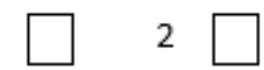

3

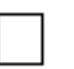

4

6.- Es necesario formar sobre el control de plagas.

1
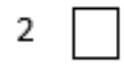

3

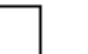

4

7.- Es necesario formar sobre la biodiversidad que podemos encontrar en el HED.

1
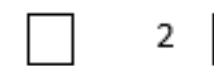

3

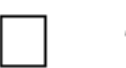

8.- Es necesario formar sobre la memoria biocultural: saberes y sabores.

1

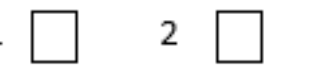

3

4

9.- Es necesario formar sobre planificación y gestión de las tareas del HED.

1
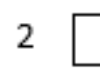

3

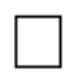

4

10.- Es necesario formar sobre la implementación de actividades en el HED.

1

$$
2
$$

3

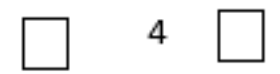

11.- Es necesario formar sobre cómo abordar los elementos transversales que se pueden trabajar en el HED.

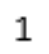

1
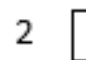

3

12.- Es necesario formar sobre los contenidos del área de Ciencias de la Naturaleza que se pueden trabajar con el HED.

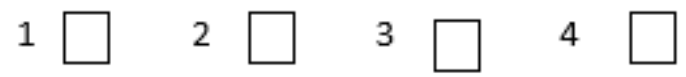

13.- Es necesario formar sobre cómo abordar los contenidos del resto de áreas del currículo de Primaria que se pueden trabajar con el HED.
1
2
3
4

14.- Es necesario formar sobre las ventajas del uso del HED como Recurso Educativo.

1

2

3

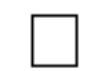

4 
15.- Es necesario formar sobre los inconvenientes del uso del HED como Recurso Educativo.

1

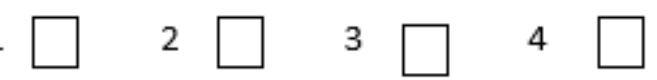

16.- Es necesario formar sobre el HED y los principios de la Agroecología.

1

2

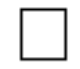

3

4

GRACIAS POR SU RELEVANTE COLABORACIÓN 
Anexo II. Cuestionario inicial y final del alumnado del Grado de Educación Primaria sobre opiniones, intereses y necesidades formativas acerca de los HED.

\section{CUESTIONARIO INICIAL Y FINAL ESTUDIANTES: EL HUERTO ESCOLAR ECOLÓGIO (HED) COMO RECURSO EDUCATIVO}

Este curso vamos a realizar una investigación sobre la formación necesaria del alumnado de la mención de Recursos de $4^{\circ}$ Grado en Primaria para el DISEÑO, USO y MANEJO del HED como RECURSO EDUCATIVO.

Para conocer cuál es vuestra formación inicial y así poder realizar un diseño adecuado a vuestras necesidades os pido vuestra colaboración mediante la cumplimentación de este cuestionario basado en la opinión y experiencia como futuros maestro/a sobre este recurso educativo, el HED.

Este cuestionario se compone de cuatro partes, una relativa a datos sociométricos y vuestra experiencia con HED; otra relativa a vuestra experiencia sobre los conocimientos didácticos que poseéis sobre este recurso, una tercera parte está relacionada con vuestros conocimientos sobre el uso, mantenimiento y gestión del HED como recurso educativo y por último, la cuarta parte recaba vuestra opinión respecto a qué conocimientos son necesarios en la formación de los futuros maestros/as para el uso de este recurso educativo. Cumplimentarlo no os llevará más de 10 minutos, gracias por vuestra colaboración.

CUESTIONARIO ALUMNOS/AS. EXPERIENCIA Y OPINIÓN SOBRE EL RECURSO HED. Marque con una $\mathbf{X}$ la casilla correspondiente o cumplimente la información solicitada.

\section{$1^{\text {a }}$ PARTE. DATOS SOCIOMÉTRICOS}

Nombre y apellidos:

Edad

\section{2ªRTE. CONOCIMIENTOS DIDÁCTICOS}

No 1 ¿Ha utilizado alguna vez el recurso educativo HED? $\square$ SI $\square$ NO

No 2 ¿Considera que el HED es un recurso educativo útil en Educación Primaria? $\square$ SI $\square$ NO

$\mathrm{N}^{\circ}$ 3. ¿Conoce alguna ventaja educativa del uso del recurso HED como recurso? $\square \mathrm{SI} \square$ NO

$\mathrm{N}^{\circ}$ 4. ¿Conoce algún inconveniente del uso del recurso HED como recurso?

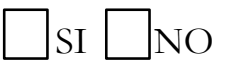

$\mathrm{N}^{\circ}$ 5.- ¿Ha recibido formación sobre el diseño, uso y mantenimiento del HED como recurso educativo? SI $\mathrm{NO}$

$\mathrm{N}^{\mathrm{o}}$ 6.- Considera que la formación que usted ha recibido es suficiente para el diseño, uso y manejo del HED como recurso educativo.

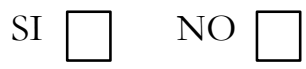

\section{$3^{\text {a }}$ PARTE. USO MANTENIMIENTO Y GESTIÓN DEL HED.}

$\mathrm{N}^{\circ}$ 1. ¿Conoce algunos de los cultivos que se pueden plantar en un HED según se trate de la plantación de otoño o de la plantación de primavera?

$\square$ SI NO $\square$ 
$\mathrm{N}^{\mathrm{o}} 2$ ¿Conoce algunas de las plantas no deseadas que hay que controlar o eliminar en un HED?

$\square$ SI NO

$\mathrm{N}^{\mathrm{o}} 3$ ¿Conoce cómo se obtiene compost?

$\square$ SI NO $\square$

$\mathrm{N}^{\circ} 4$ ¿Conoce cómo se gestiona un banco de semillas para un HED?

$\square$ SI NO $\square$

$\mathrm{N}^{\circ} 5$ ¿Conoce alguna plaga habitual en los HED?

$\square$ SI NO $\square$

$\mathrm{N}^{\circ} 6$ ¿Conoce los principios de la agroecología?

$\square$ SI NO

$\mathrm{N}^{\circ} 7$ ¿Sabe en qué consiste la soberanía alimentaria?

$\square$ SI NO $\square$

$4^{a}$ PARTE. OPINIÓN SOBRE LOS CONTENIDOS NECESARIOS EN LA FORMACIÓN DE LOS FUTUROS MAESTROS/AS SOBRE EL USO, GESTIÓN Y MATENIMIENTO DEL HED COMO RECURSO EDUCATIVO.

Señale desde 1 a 4 la opción que usted considera más adecuada utilizando los siguientes valores: 1 . No es necesario. 2. Es poco necesario. 3. Es necesario y 4. Es muy necesario.

$\mathrm{N}^{\circ}$ 1.- Es necesario formar respecto a los tipos de HED y sus diseños

1

2

3

4

$\mathrm{N}^{\mathrm{o}}$ 2.- Es necesario formar respecto a los tipos de cultivos a plantar.

1

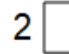

3

4

$\mathrm{N}^{\circ}$ 3.- Es necesario formar en cuanto a su limpieza, control de plantas no deseadas.

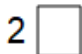

3

4

$\mathrm{N}^{o}$ 4. Es necesario formar en la realización de compost (compostaje o vermicompostaje)

1

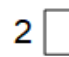

$3 \square$

4

$\mathrm{N}^{\circ}$ 5.- Es necesario formar sobre las semillas a utilizar.

1

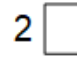

3

4

$\mathrm{N}^{\circ}$ 6.- Es necesario formar sobre el control de plagas.

1

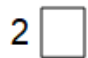

3

4

$\mathrm{N}^{\mathrm{o}}$ 7.- Es necesario formar sobre la biodiversidad que podemos encontrar en el HED.

1

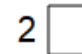

3

4 
$\mathrm{N}^{\circ}$ 8.- Es necesario formar sobre la memoria biocultural: saberes y sabores.

1

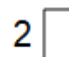

3

4

$\mathrm{N}^{\circ}$ 9.- Es necesario formar sobre planificación y gestión de las tareas del HED.

1

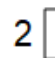

3

4

$\mathrm{N}^{\circ}$ 10.- Es necesario formar sobre la implementación de actividades en el HED.

2

3

4

$\mathrm{N}^{\circ}$ 11.- Es necesario formar sobre cómo abordar los elementos transversales.

1

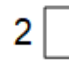

3

4

$\mathrm{N}^{\mathrm{o}}$ 12.- Es necesario formar sobre cómo abordar aquellos contenidos del área de Ciencias de la Naturaleza utilizando el HED como recurso educativo.

1

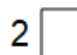

3

4

$\mathrm{N}^{\mathrm{o}}$ 13.- Es necesario formar sobre cómo abordar contenidos del resto de áreas del currículo de Primaria utilizando el HED como recurso educativo.

1
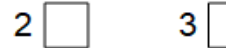

4

$\mathrm{N}^{\mathrm{o}}$ 14.- Es necesario formar sobre las ventajas del uso del HED como Recurso Educativo.

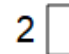

3

4

$\mathrm{N}^{\circ}$ 15.- Es necesario formar sobre los inconvenientes del uso del HED como Recurso Educativo

1

2

3

4

$\mathrm{N}^{\circ}$ 16.- Es necesario formar sobre el HED y los principios de la Agroecología.

1

2

3

4

GRACIAS POR SU RELEVANTE COLABORACIÓN 